\title{
The pharmacokinetics of dexmedetomidine during long-term infusion in critically ill pediatric patients. A Bayesian approach with informative priors
}

\author{
Paweł Wiczling ${ }^{3}$ - Alicja Bartkowska-Śniatkowska ${ }^{1}$ - Oliwia Szerkus ${ }^{3}$. \\ Danuta Siluk $^{3}$ - Jowita Rosada-Kurasińska ${ }^{1}$ - Justyna Warzybok ${ }^{2}$. \\ Agnieszka Borsuk $^{3} \cdot$ Roman Kaliszan $^{3} \cdot$ Edmund Grześkowiak $^{2} \cdot$ Agnieszka Bienert $^{2}$
}

Received: 15 February 2016/ Accepted: 6 May 2016/Published online: 24 May 2016

(C) The Author(s) 2016. This article is published with open access at Springerlink.com

\begin{abstract}
The purpose of this study was to assess the pharmacokinetics of dexmedetomidine in the ICU settings during the prolonged infusion and to compare it with the existing literature data using the Bayesian population modeling with literature-based informative priors. Thirty-eight patients were included in the analysis with concentration measurements obtained at two occasions: first from 0 to $24 \mathrm{~h}$ after infusion initiation and second from 0 to $8 \mathrm{~h}$ after infusion end. Data analysis was conducted using WinBUGS software. The prior information on dexmedetomidine pharmacokinetics was elicited from the literature study pooling results from a relatively large group of 95 children. A two compartment PK model, with allometrically scaled parameters, maturation of clearance and t-student residual distribution on a log-scale was used to describe the data. The incorporation of time-dependent (different between two occasions) PK parameters improved the model. It was observed that volume of distribution is 1.5fold higher during the second occasion. There was also an evidence of increased (1.3-fold) clearance for the second
\end{abstract}

Electronic supplementary material The online version of this article (doi:10.1007/s10928-016-9474-0) contains supplementary material, which is available to authorized users.

Paweł Wiczling

wiczling@gumed.edu.pl

$\triangle$ Alicja Bartkowska-Śniatkowska

asniatko@ump.edu.pl

1 Department of Pediatric Anesthesiology and Intensive Therapy, Poznan University of Medical Sciences, Szpitalna Street 27/33, 60572 Poznan, Poland

2 Department of Clinical Pharmacy and Biopharmacy, Poznan University of Medical Sciences, Poznan, Poland

3 Department of Biopharmaceutics and Pharmacodynamics, Medical University of Gdansk, Gdansk, Poland occasion with posterior probability equal to $62 \%$. This work demonstrated the usefulness of Bayesian modeling with informative priors in analyzing pharmacokinetic data and comparing it with existing literature knowledge.

Keywords Dexmedetomidine · WinBUGS · Population pharmacokinetics $\cdot$ Informative priors

\section{Introduction}

The dosage of most drugs in children is based on extrapolation of pharmacokinetic and pharmacodynamic data obtained from adults using body weight scaling, age and occasionally other patient's characteristics, such us gene polymorphism [1]. Without taking into account the degree of maturation of various organ in children and neonates in pharmacokinetic extrapolations, over or under dosing might occur, which consequently might lead to serious complications, side effects and lack of expected therapeutic effects $[2,3]$. Therefore, the identification of inter-individual differences directly or indirectly affecting pharmacokinetics (PK) of drugs, is very important for selecting the individual and the optimal dose, especially in children under severe conditions. It especially applies to new drugs such as dexmedetomidine (DEX), for which there is a relatively small number of studies performed on special population, like that from pediatric intensive care units (PICU).

DEX as a potent, highly selective and more specific $\alpha 2-$ adrenoceptor agonist [4] has become an interesting alternative drug for so far widely used benzodiazepines during general anaesthesia and sedation in intensive care. Its unique characteristics makes it an $\alpha$-adrenoceptor agonist with $\alpha 2: \alpha 1$ selectivity ratio of $1600: 1$, especially for the $\alpha 2 \mathrm{~A}$ subtype, providing increased sedation, anxiolysis 
and analgesia without breathing depression. Dexmedetomidine is metabolized in liver with hepatic extraction ratio of 0.71 and the mean elimination half-life of about 2-2.5 h. Glucuronidation is the process, which poses one-third of metabolism. The other pathways involve multiple cytochrome P450 enzymes, especially CYP2A6, but also CYP1A2, CYP2C19, CYP2D6, and CYP2E1. Approximately $90 \%$ of administered human drug dose is excreted as metabolites in urine, and $10 \%$ in feces [5]. Its influence on brain and spinal cord, mainly via locus coreuleus (LC), provides effects, which are different from those produced by other standard drugs (e.g. clonidine). It diminishes impaired sleep deprivation or poor sleep quality, especially during long-term sedation. DEX, in contrast to benzodiazepines, does not disrupt REM sleep and more closely resembles natural non-REM phase, as well as regulates the circadian rhythm by shifting sleep from day to the night [610]. DEX has been effectively used in patients more often presenting agitation and developing higher risk of delirium during conventional sedation, allowing shortening of mechanical ventilation and thus, stay in the PICU [11-14]. Despite increasing number of clinical experiences, pharmacokinetic and pharmacodynamic characteristics of DEX still remain unclear, forcing the need for further research, especially in the youngest patients $[15,16]$.

The data obtained from routine clinical monitoring are challenging in terms of interpretation and are often collected in not perfectly-controlled experiments. For such data, using a full conditional Bayesian modeling approach with prior's information is very appealing. In this work we explore the use of informative priors to analyze the data obtained during routine hospitalization of children in an intensive care unit and to identify differences between our study and the currently established knowledge on DEX pharmacokinetics. The analysis consisted of several steps (1) elucidation of prior's information on the type of model and its parameters from the literature, (2) development of a pharmacokinetic model, (3) determination of covariate relationship which could explain inter-individual and intraindividual differences in drug PK, and (4) identification of differences between literature-described patients and those enrolled in this study.

The possibility of using informative priors is a particular strength of the Bayesian framework [17]. During this type of analysis the priors and the newly collected data are appropriately weighted yielding a posteriori distribution of parameters and predictions that provide logically consistent inference conditional on all the explicitly stated assumptions, such as structural model and priors. Nevertheless, the Bayesian inference using Markov chain Monte Carlo (MCMC) algorithm is not very popular, as generally it is computer intensive. There are only few population pharmacokinetic analyses published which used WinBUGS
[18-22]. Informative prior (with relatively high precision) was rarely used [23-25].

\section{Materials and methods}

\section{Patients}

In our study, DEX was used in addition to the standard algorithm of sedation applicable in our PICU which consists of sufentanyl and midazolam administration [26, 27]. Similarly, sedation monitoring was also carried out by the Cook Scale, which has been routinely used by experienced and trained nurses' team in our department [28]. This scale was originally adapted from Glasgow Coma Scale (GCS) based on the assessment of four reactions, such as eye opening, cough reflex, respiration and motor activity, in response to the stimulus, ranging from minimum of 4 (deep sedation) to maximum of 18 points (awakening). Decision on the addition of DEX to the standard sedation and analgesia was made by a doctor (paediatric intensivist) in order to prevent delirium and/or facilitate awakening of patient. The pediatric risk of mortality (PRISM) score was determined for all patients in the admission to PICU. It is a physiologically based score used to quantify physiologic status, and when combined with other independent variables, it can compute expected mortality risk and expected morbidity risk [29].

Thirty-eight patients were enrolled in the study. Informed consent was obtained from the parents or legal representatives according to the approval of the Institutional Bioethics Committee (no 276/12). Exclusion criteria included the following factors: age $>18$ years, known allergy to DEX, previous administration of neuromuscular blocking agents and severe renal and/or hepatic insufficiency with serum bilirubin and creatinine levels twofold higher than upper limits of normal reference values.

Continuous intravenous infusion of DEX was routinely initiated at the rate of $0.8 \mu \mathrm{g} / \mathrm{kg} / \mathrm{h}$. Among the patients requiring mechanical ventilation, infusion of DEX was gradually increased or decreased by $0.2 \mu \mathrm{g} / \mathrm{kg} / \mathrm{h}$ to maintain the level of sedation between 7 and 14 points in the Cook Scale. Maximum dose of DEX was $1.4 \mu \mathrm{g} / \mathrm{kg} / \mathrm{h}$. Otherwise, if the doctor decides that the patient could be awakened, DEX was decreased by $0.2 \mu \mathrm{g} / \mathrm{kg} / \mathrm{h}$ to its minimum dose, till the end of infusion. At the same time, the doses of sufentanyl and midazolam were alternatively reduced to $0.01-0.05 \mu \mathrm{g} / \mathrm{kg} / \mathrm{h}$ and $0.01-0.1 \mathrm{mg} / \mathrm{kg} / \mathrm{h}$, respectively, to obtain adequate sedation while maintaining spontaneous respiration. Sedation for each patient included in the study was adjusted individually considering the clinical criteria of intensive therapy. 
Blood samples for PK assessment $(2.0 \mathrm{~mL})$ were collected from the arterial catheter according to the protocol of the study. The first blood sample was collected just before the initiation of DEX infusion, and further samples were collected at $1,4,8,12,16,20$, and $24 \mathrm{~h}$ during the first day (occasion 1). When DEX infusion was stopped, blood samples were collected just before the cessation, and then, at 5, 10, 20 min and 1, 2, 4 and $6 \mathrm{~h}$ after the infusion end (occasion 2). All blood samples were centrifuged immediately after collection, and plasma was stored at $-80{ }^{\circ} \mathrm{C}$ until analysis.

\section{Analytical methods}

Analytical method description was presented in detail elsewhere [30]. Briefly, extraction of DEX from $500 \mu \mathrm{l}$ plasma was performed with the use of solid-phase extraction Bond-Elut Plexa cartridges $(30 \mathrm{mg}, 1 \mathrm{ml}$, Agilent Technologies, Inc., Palo Alto, CA, USA). Extracted samples were evaporated to dryness at a miVac Quattro Sample Concentrator (Genevac, Suffolk, UK), reconstituted with $100 \mu \mathrm{l}$ of methanol, and injected onto the chromatographic system. Analyses were performed with the use of an 1260 HPLC system (Agilent Technologies, Inc., Palo Alto, CA, USA) composed of degasser (G1322A), binary pump (G1312B0, thermostated autosampler (G1329B) coupled with triple-quadrupole mass spectrometer (6430) with electrospray ionization source (ESI). The separation was carried out using a Zorbax Eclipse Plus C18 $(4.6 \times 100 \mathrm{~mm}, \quad 3.5 \mu \mathrm{m}$, Agilent Technologies). The mobile phase, pumped at a flow rate of $0.5 \mathrm{ml} / \mathrm{min}$, was composed of a mixture of water and methanol with addition of 0.1 formic acid $(2: 8, \mathrm{v} / \mathrm{v})$. The analyses were performed with the use of detomidine as an internal standard (IS). The total analysis time was 3 min.

The software used for data acquisition and processing was MassHunter Workstation v. B.07.01. (Agilent Technologies, Inc., Palo Alto, CA, USA). Ions were detected using multiple reaction monitoring (MRM) acquisition mode at the following mass transitions: $\mathrm{m} / \mathrm{z} 201 \rightarrow 95$ (quantifier), $\mathrm{m} / \mathrm{z} 201 \rightarrow 68$ (qualifier) for $\mathrm{DEX}$ and $\mathrm{m} / \mathrm{z}$ $187 \rightarrow 81$ for IS. The quantification of the analyte concentration was based on area peak ratios of DEX over IS. Mass spectrometry parameters: fragmentor voltage, collision energies and ESI parameters (gas flow, nebulizer pressure, drying gas temperature and capillary voltage) are listed in supplementary material.

The developed and optimized method was validated following the guidelines of the United States Food and Drug Administration (FDA) for bioanalytical method validation [31]. It was validated in terms of linearity, specificity, lower limit of quantification, recovery, intra- and inter-day precision and accuracy, analyte stability during the sample processing and storage as well as in terms of matrix effects; all the parameters met the FDA bioanalytical requirements. Each analytical sequence included double blank sample, blank sample, calibration standards (5, $10,50,100,500,1000,2500 \mathrm{ng} / \mathrm{ml}$ ) and quality controls $(20,200,2000 \mathrm{ng} / \mathrm{ml})$. The intra- and inter-day precision ranged between 5 and 7.4 RSD, respectively, and accuracy of the assay reached an average of 101.6 and 103.0, for intra- and inter-day tests. LOD, based on $\mathrm{S} / \mathrm{N}$ ratio 3, equaled $1.5 \mathrm{pg} / \mathrm{ml}$.

\section{PK model development}

Population modeling was performed using WinBUGS 1.4.3. The BUGS language interface was implemented using WBDev and BlackBox 1.5 compiler as described elsewhere [32]. Data management, launching WinBUGS, and analysis of the MCMC samples were done in Matlab Software (Version 8.1; The MathWorks, Natick, MA, USA) using the MatBUGS interface. Three MCMC chains of 6000 iterations were simulated. The first 3000 iterations of each chain were discarded and every 3rd sample was retained. Thus 3000 MCMC samples were used for subsequent analyses. Model convergence was assessed by Gelman-Rubin diagnostics available in WinBUGS. The MCMC chains were assumed to have reached the stationary distribution if Gelman-Rubin values were less than 1.2 for all parameters. Furthermore, the trace history of MCMC samples for all chains was examined visually for all parameters, for which 'fuzzy caterpillar' suggests that MCMC chains had reached a stationary distribution [17]. All the codes are available in the Supplementary Materials. Model selection was based on deviance information criterium (DIC), which is the mean of the deviance distribution $(-2 \log$ likelihood) plus penalty for the effective number of parameters in the model.

The DEX plasma concentrations were characterized by a two-compartment model. The following equation were used:

$V_{p} \frac{d C_{p}}{d t}=R_{0}(t)-C L C_{p}-Q C_{p}+Q C_{T} \quad C_{p}(0)=0$

$V_{T} \frac{d C_{T}}{d t}=Q C_{P}-Q C_{T} \quad C_{T}(0)=0$

where $C_{P}, C_{T}$ denotes concentrations of DEX in central and peripheral compartments. The model was parameterized with volume and clearance terms. The $V_{P}, V_{T}$ denote volumes of distribution of the respective compartments, $C L$ denotes metabolic clearance of DEX and $Q$ denotes the inter-compartmental clearance. The $R_{0}$ denotes the infusion rate and all extra boluses that were administered to a patient. All tested models were parameterized in terms of the natural $\log$ of the parameter values (i.e. $\ln (C L)$ ). 
Inter-individual variability (IIV) for all PK parameters was modeled assuming log normal distribution:

$\ln P_{i}=\ln \theta_{P}+\eta_{P, i}$

where $P_{i}$ are PK parameter for the $i$ th subjects, $\theta_{P}$ is the typical value of this parameter in the population, and $\eta_{P}$ is a random effect for that parameter with mean 0 and variance $\omega_{P}^{2}$.

Any $j$ th observation of DEX concentration for the $i$ th individual, $C_{P i j}$ at time $t_{j}$, was defined on a log scale by:

$\log C_{P, i j}=\ln C_{P}+\varepsilon_{C, i j}$

where $C_{P}$ is defined by the basic structural model (Eq. (1)) and represents the additive (on a log scale) random error for PK measurements. It was assumed that is t-distributed with mean 0 and scale of the t-distribution denoted by $\sigma_{C}$ and degrees of freedom (or normality parameter) $v$ to account for some outlying measurements present in the dataset.

The effect of body size on all the volume $\left(V_{C}, V_{T}\right)$ and clearance $(C L, Q)$ parameters was included based on allometric scaling as follows:

$\ln P_{i}=\ln \theta_{p}+\ln f_{P}+K \ln \left[\frac{B W_{i}}{70}\right]+\eta_{P, i}$

where $P_{i}$ denotes the individual value of volume and clearance term; the population estimates of volume and clearance terms, $B W_{i}$ the individual body weight, where 70 is a typical body weight of adult patients, and $K$ is the exponent equal to 0.75 for clearance and 1 for distribution volumes [33]. All parameters were different between occasions with a fractional change $f_{P}$ for occasion $2\left(f_{P}=1\right.$ for occasion 1). Additionally, for clearance an age-dependent maturation was included:

$$
\begin{aligned}
\ln C L_{i}= & \ln \theta_{C L}+\ln f_{P}+K \ln \left[\frac{B W_{i}}{70}\right] \\
& +\ln \left[\frac{P M A_{i}^{\text {Hill }}}{P M A_{i}^{\text {Hill }}+T E_{50}^{\text {Hill }}}\right]+\eta_{C L, i}
\end{aligned}
$$

where $C L_{i}$ denotes the individual value of clearance; $P M A_{i}$ the individual postmenstrual age of the patient; $T E_{50}$, and Hill are Hill equation parameter reflecting the slope and the degree of clearance maturation. Following the WinBUGS parameterization [17, 23] (uncertainty is described as a precision, which is an inverse of variance), the stochastic parts of the model can be represented as:

$\varepsilon_{i j} \sim t\left(0, \sigma_{C}^{-2}, v\right)$

$\log P_{i} \sim \operatorname{MVN}\left(\ln \theta_{P}, \Omega^{-1}\right)$

where $\mathrm{t}$ denotes $\mathrm{t}$-distribution and $\mathrm{MNV}$ is multivariate normal distribution. The model for the priors is as follows:
$\sigma_{C} \sim \operatorname{Uniform}(0.001,1000)$

$v \sim 1+\operatorname{Exponential}(0.1)$

$\log \theta \sim \operatorname{MVN}\left(\ln \bar{\mu}, \sum^{-1}\right)$

$\Omega^{-1} \sim \operatorname{Wishart}\left(\rho \Omega_{0}, \rho\right)$

The priors consisted of the vector of hyperprior population mean parameters, $\bar{\mu}$, its precision, the expected intrasubject variance $\Omega_{0}$ and its precision given by Wishart distribution degrees of freedom $\rho$. For the residual error model sigma (scale of the t-distribution) was assumed to follow a uniform distribution and $v$ (normality parameter) was constrained to be larger than one and following an exponential distribution.

\section{Prior selection}

The informative priors for the typical value of PK parameters and their inter-individual variability were elucidated from the work [34] and are presented in Table 1. The priors were obtained from the pooled analysis of four studies investigating DEX pharmacokinetics after i.v. administration to 95 children.

\section{Covariates}

The potential effect of various covariates (listed in Table 2) on DEX PK was assessed in this study in addition to the a priori assumed effects of body weight and age on PK parameters. The potential covariate relationships were assessed by plotting the mean a posteriori values of the PK parameters against the available covariates (weight, age, sex, dose, infusion duration, and PRISM) to identify their potential effects. If any relationship was found, it was described by means of linear regression or power model (allometric relationship). The categorical covariates were included into the model based on indicator variables.

Additionally the difference in PK parameters between the two occasions was tested during the model building process. The fraction parameters for all PK parameters were assumed to be equal to 1 ( 0 on a $\log$ scale) with precision

$\log f_{P} \sim N\left(\log 1, \sigma_{f_{P}}^{-2}\right)$

The selected values of $\sigma_{f P}$ which spanned within a range from 0.01 to 0.6 , were compared. The smallest value corresponds to a priori assumption of the lack of difference between the two occasions, and the latter one corresponds to a vague prior on the fraction parameter. The models with the lowest deviance information criterion, the best predictive performance, and the most conservative (with the 
Table 1 Prior distributions for $\theta$ and $\Omega$ as derived from [34]

\begin{tabular}{|c|c|c|c|c|c|c|c|c|c|}
\hline \multirow[t]{2}{*}{ Parameter, units } & \multirow[t]{2}{*}{ Description } & & \multicolumn{3}{|c|}{ Reported } & \multicolumn{4}{|c|}{ Used in WinBUGS } \\
\hline & & & $\bar{\mu}$ & $\% \mathrm{SE}$ & $95 \% \mathrm{CI}$ & $\ln 95 \% \mathrm{CI}$ & $\log \bar{\mu}$ & $\Sigma$ for $\ln \bar{\mu}^{\mathrm{a}}$ & $\Sigma^{-1}$ for $\ln \bar{\mu}$ \\
\hline \multicolumn{10}{|l|}{ Fixed effects } \\
\hline $\mathrm{CL}, \mathrm{L} / \mathrm{h} 70 \mathrm{~kg}^{-1}$ & Total clearance & & 42.1 & 4.4 & $(38.7-45.8)$ & $(3.7-3.8)$ & 3.7 & 0.00185 & 542 \\
\hline $\mathrm{Q}, \mathrm{L} / \mathrm{h} 70 \mathrm{~kg}^{-1}$ & Distribution clearance & & 78.3 & 14.4 & $(50.7-98.4)$ & $(3.9-4.6)$ & 4.3 & 0.02862 & 34.9 \\
\hline $\mathrm{V}_{1}, \mathrm{~L} 70 \mathrm{~kg}^{-1}$ & Volume of distribution of central comp & rtment & 56.3 & 8.7 & $(44.5-67.4)$ & $(3.8-4.2)$ & 4.0 & 0.01122 & 89.2 \\
\hline $\mathrm{V}_{2}, \mathrm{~L} 70 \mathrm{~kg}^{-1}$ & $\begin{array}{l}\text { Volume of distribution of peripheral } \\
\text { compartment }\end{array}$ & & 69 & 8.2 & $(57.5-80.3)$ & $(4.1-4.4)$ & 4.2 & 0.00726 & 138 \\
\hline $\mathrm{TE}_{50}$, weeks & $\begin{array}{l}\text { Age at which clearance is } 50 \% \text { of } \\
\text { adult value }\end{array}$ & & 44.5 & 6.9 & $(36.8-50.3)$ & $(3.6-3.9)$ & 3.8 & 0.00636 & 157 \\
\hline Hill & Slope of clearance maturation & & 2.56 & 17.6 & $(1.65-3.78)$ & $(0.5-1.3)$ & 0.9 & 0.04472 & 22.4 \\
\hline \multirow[t]{2}{*}{ Parameter, units } & & \multirow{2}{*}{\multicolumn{3}{|c|}{ Description }} & \multicolumn{2}{|c|}{ Reported } & \multicolumn{3}{|c|}{ Used in WinBUGS } \\
\hline & & & & & $\% \mathrm{CV}$ & & $\Omega_{0}^{\mathrm{b}}$ & & $\Omega_{0}^{-1}$ \\
\hline \multicolumn{10}{|c|}{ Between subject variability (diagonal elements) } \\
\hline \multicolumn{2}{|l|}{ CL } & \multicolumn{3}{|c|}{ Variability for $\mathrm{CL}$} & \multicolumn{2}{|l|}{30.9} & \multicolumn{2}{|c|}{0.091} & 11.0 \\
\hline \multicolumn{2}{|l|}{ Q } & \multicolumn{3}{|c|}{ Variability for Q } & \multicolumn{2}{|l|}{37} & \multicolumn{2}{|l|}{0.13} & 7.79 \\
\hline \multicolumn{2}{|l|}{$\mathrm{V}_{1}$} & \multicolumn{3}{|c|}{ Variability for $\mathrm{V}_{1}$} & \multicolumn{2}{|l|}{61.3} & \multicolumn{2}{|l|}{0.32} & 3.13 \\
\hline \multirow[t]{2}{*}{$\mathrm{V}_{2}$} & & \multirow{2}{*}{\multicolumn{3}{|c|}{ Variability for $\mathrm{V}_{2}$}} & \multirow{2}{*}{\multicolumn{2}{|c|}{47}} & \multicolumn{2}{|l|}{0.20} & 5.01 \\
\hline & & & & & & & $\rho^{c}$ & & 30 \\
\hline
\end{tabular}

For $\Sigma, \Sigma^{-1}, \Omega_{0}$ and $\Omega_{0}^{-1}$ only diagonal elements are provided (the off-diagonal elements are zero)

a Calculated based on (97.5th-2.5th)/2/1.96, where 2.5th and 97.5th are $95 \%$ confidence intervals (on a log scale) from bootstrap

b Calculated based on $\ln \left((\% \mathrm{CV} / 100)^{2}+1\right)$

c The value of $\rho$ was determined empirically for the variance-covariance matrix by simulating from the Wishart distribution in MATLAB (Version 6.5, The MathWorks, Natick, MA) to ensure $25 \%$ of variability in $\Omega_{0}$ parameters. The standard errors for $\% \mathrm{CV}$ were not reported in the original article

Table 2 Demographic characteristic of patients

\begin{tabular}{ll}
\hline Parameter, unit & Median [range] or number \\
\hline Age, months & $70[1.4-188.6]$ \\
Weight, kg & $18.5[4.7-60]$ \\
Dose, $\mu \mathrm{g}$ & $1153.8[248.8-4732.2]$ \\
Infusion duration, h & $97.3[45.0-229.2]$ \\
Pediatric risk of mortality (PRISM) & $0.5[0-11]$ \\
Male/female & $23 / 15$
\end{tabular}

lowest $\left.\sigma_{f P}\right)$, were selected as final. A clinically significant difference in the fraction parameters was claimed when $20 \%$ difference was observed.

\section{Posterior predictive check and model assessment}

The model performance was assessed by means of a posteriori predictive check. The plots were drawn from individual a posteriori PK predictions. In this study the 10th, 50th and 90th percentiles were used to summarize the data and model predictions. This graph resembles the classical visual predictive check (VPC) and enables the comparison between confidence intervals obtained from prediction and the observed data over time. When the corresponding percentile from the observed data falls outside the $95 \%$ confidence interval derived from predictions, there is an evidence of model misspecification. Since the PK data deviated from nominal times to some extent, binning across time was performed. Next, the prediction error (PE) was calculated for each measurement as PE $=100$ (measured - population predicted)/population predicted, and was summarized as a median for each individual. The median prediction error (MDPE) and median absolute prediction error (MDAPE) were calculated according to the formulas:

$$
\begin{array}{r}
\mathrm{MDPE}=\operatorname{median}\left(P E_{1}, P E_{2}, \ldots P E_{n}\right) \\
\mathrm{MDAPE}=\operatorname{median}\left(\left|P E_{1}\right|,\left|P E_{2}\right|, \ldots\left|P E_{n}\right|\right)
\end{array}
$$

where $n$ denotes the number of individuals. MDPE reflects the bias of the model, whereas MDAPE reflects the inaccuracy of the prediction. 


\section{Results}

This analysis was based on the concentration-time profiles of DEX collected from 38 PICU children. Thirty-eight patients, with median (range) age of 70 months (1.4-188.6) diagnosed in our PICU with acute respiratory failure $(\mathrm{n}=18)$, severe sepsis or septic shock $(\mathrm{n}=10)$, multiple or brain trauma $(\mathrm{n}=8)$ and acute cardiac insufficiency $(n=2)$, were enrolled in the study. Table 2 lists the patients' demographic, clinical laboratory and vital signs characteristics. The available data consisted of 470 DEX concentration measurements measured at two occasions as presented in Fig. 1. In our data few outlying measurements were evident (with concentrations a few-fold higher or lower than average) and so, a robust residual error model was needed. We decided to use a t-distribution on a logtransformed concentrations.

The modeling was done using Bayesian inference with informative priors. It was required due to small number of patients and observational nature of the study design, which limited the ability to precisely estimate all PK parameters. The model-building process started with a twocompartment model, for which after implementation of an allometric scaling to all clearance and volume of distribution parameters, age maturation of clearance turned out to be insufficient to describe our data. Without occasion as a covariate there was an evidence of miss-prediction in the initial phase after infusion cessation as reflected by MDPE
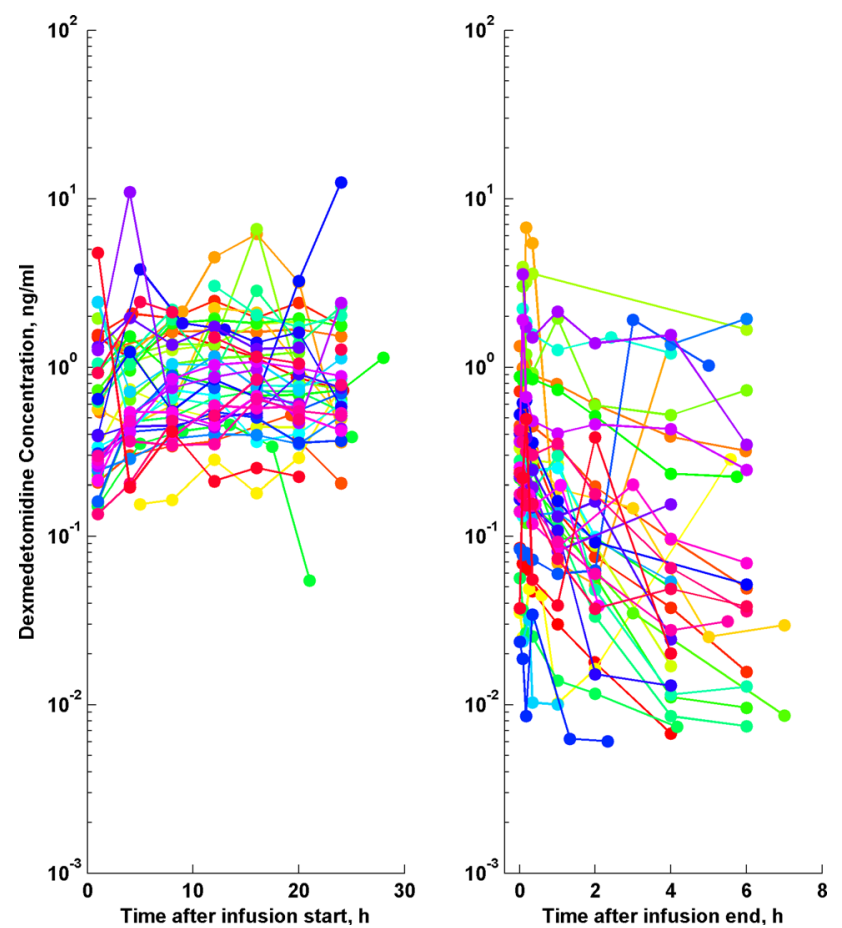

Fig. 1 The individual dexmedetomidine concentration-time profiles
$(-11.50)$ and posterior predictive check (data not shown). The inclusion of fractional change for all PK parameters improved the model as demonstrated by lower DIC value (DIC changed from 840.86 to $825.68, \Delta \mathrm{DIC}=15.176$ ) and reduced the bias (MDPE decreased from -11.50 to -2.2 ) observed initially in predictive check plots. The supplementary materials present the influence of prior precision on the posterior distribution of parameters. The $\sigma_{f P}=0.2$ represents the most parsimonious choice as further increase in its values did not improve the accuracy of model predictions.

The goodness-of-fit plots of the final PK model are shown in supplemental materials. The individual predictions are very close to that obtained from the experimental data, indicating good performance of the model, which is also confirmed by other goodness-of-fit diagnostic plots. The posterior predictive check for the DEX concentration was used to assess the simulation properties of the model presented in Fig. 2. Both the central tendency of the data and the variability at a particular sampling time were recaptured well. There are no major misspecifications in that graph.

Table 3 provides the means and credibility interval for all PK parameters. The typical value of the volume of the central compartment $\left(V_{1}\right)$ scaled to $70 \mathrm{~kg}$ was $52.0 \mathrm{~L}$, whereas the volume of the peripheral compartment was slightly higher $\left(V_{2}=70.4 \mathrm{~L}\right)$. The typical systemic clearance $(C L)$ of DEX and the distribution clearance $(Q)$ were 41.6 and $56.8 \mathrm{~L} / \mathrm{h}$ for $70-\mathrm{kg}$ patients. The IIV was estimated for the $C L, Q$, and $V_{1}$ and $V_{2}$, for which it amounted to $56,83,152,68 \%$ and a strong correlation (0.7) between $Q$ and $V_{1}$. Those values are consistent with literature parameters in children and adults and are very close to the priors used [34]. The change between mean prior and mean posterior values was $-1.2,-27.5,-7.6,2.0,-4.5,-4.3$ for $C L, Q, V_{1}, V_{2}, T E_{50}$ and Hill, respectively.

The final model included the difference in PK parameters between two occasions as reflected by the fraction parameters $f_{P}$. The posterior probability for inclusion of the fractional effect on occasion was $>0.5$ for a $20 \%$ change in the parameters $\left(\operatorname{Pr}=0.62\right.$ for $C L$ and $\operatorname{Pr}=1.00$ for $\left.V_{1}\right)$. For other parameters the probability was lower than 0.5 . The volume of distribution and clearance was 1.5 -fold (with 5th-95th credible interval of 1.33-1.65) and 1.3-fold (with 5th-95th credible interval of 0.91-1.82) higher at the second occasion, respectively.

The children enrolled in this study exhibited a large difference in body weight ranging from 4.7 to $60 \mathrm{~kg}$. In this study the allometric scaling with theory-based exponents for all clearance and volume terms was used along with a clearance maturation model. The actual and body weighted normalized values of clearance and volume of distribution in relation to patient age are presented in Fig. 3. None of 
Fig. 2 The visual a posteriori predictive plots for final dexmedetomidine PK model. The plots show the individual prediction-based $95 \%$ confidence intervals around the 10th, 50th, and 90th percentiles of the PK data (blue areas). The corresponding percentiles from the experimental data are plotted in black color. The raw data is presented as gray closed symbol (Color figure online)
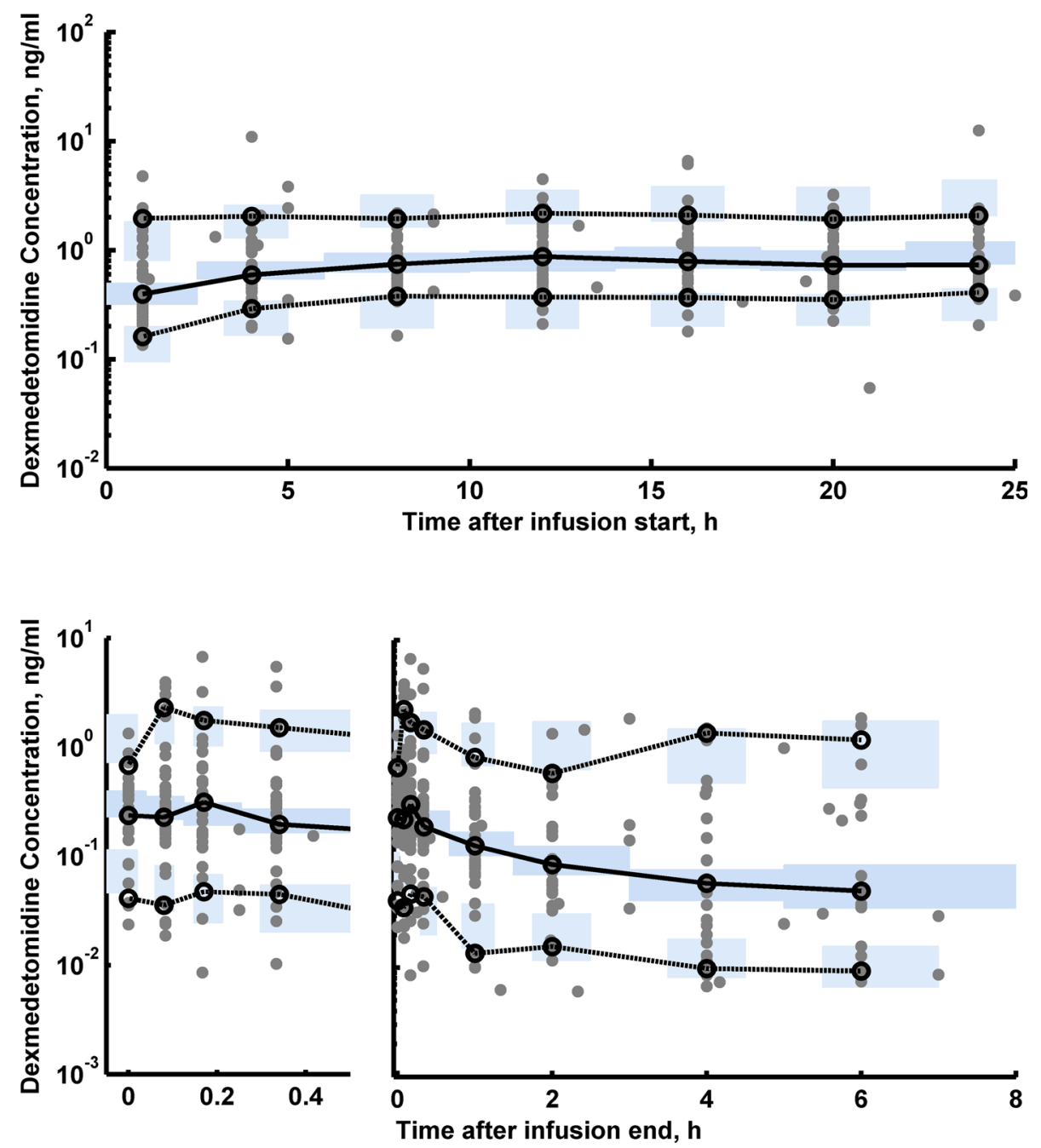

Table 3 Summary of the MCMC simulations of the marginal posterior distributions of pharmacokinetic parameters from the final model of dexmedetomidine

\begin{tabular}{lll}
\hline Parameter, unit & Description & $\theta$, Mean (90 \% HDI) \\
\hline$\theta_{\mathrm{CL}}, \mathrm{L} / \mathrm{h} 70 \mathrm{~kg}^{-1}$ & Total clearance & $41.6(39.0-44.3)$ \\
$\theta_{\mathrm{Q}}, \mathrm{L} / \mathrm{h} 70 \mathrm{~kg}^{-1}$ & Distribution clearance & $56.8(43.5-73.5)$ \\
$\theta_{\mathrm{V} 1}, \mathrm{~L} 70 \mathrm{~kg}^{-1}$ & Volume of distribution of central compartment & $52.0(43.2-59.6)$ \\
$\theta_{\mathrm{V} 2}, \mathrm{~L} 70 \mathrm{~kg}^{-1}$ & Volume of distribution of peripheral compartment & $70.4(63.0-79.8)$ \\
$\theta_{\mathrm{TE} 50}$, weeks & Age at which clearance is 50 \% of adult value & $42.5(61.7-47.8)$ \\
$\theta_{\mathrm{Hill}}$ & Slope of clearance maturation & $2.45(1.72-3.39)$ \\
$f_{\mathrm{CL}}$ & Fractional change of CL & $1.31(0.910-1.82)$ \\
$f_{\mathrm{Q}}$ & Fractional change of Q & $1.02(0.722-1.40)$ \\
$f_{\mathrm{V} 1}$ & Fractional change of V1 & $1.50(1.33-1.65)$ \\
$f_{\mathrm{V} 2}$ & Fractional change of V2 & $0.86(0.630-1.17)$ \\
\hline
\end{tabular}

HDI highest density interval the covariate (Table 2) was found to be statistically significant in this study (in addition to the a priori assumed age and body weight effects) as there is no clear relationship between them and individual PK parameter estimates.
The ETA plots (deviation of the individual estimate from the population mean in relation to covariate) for age, duration of infusion, PRISM, sex, weight are shown in supplementary materials. 

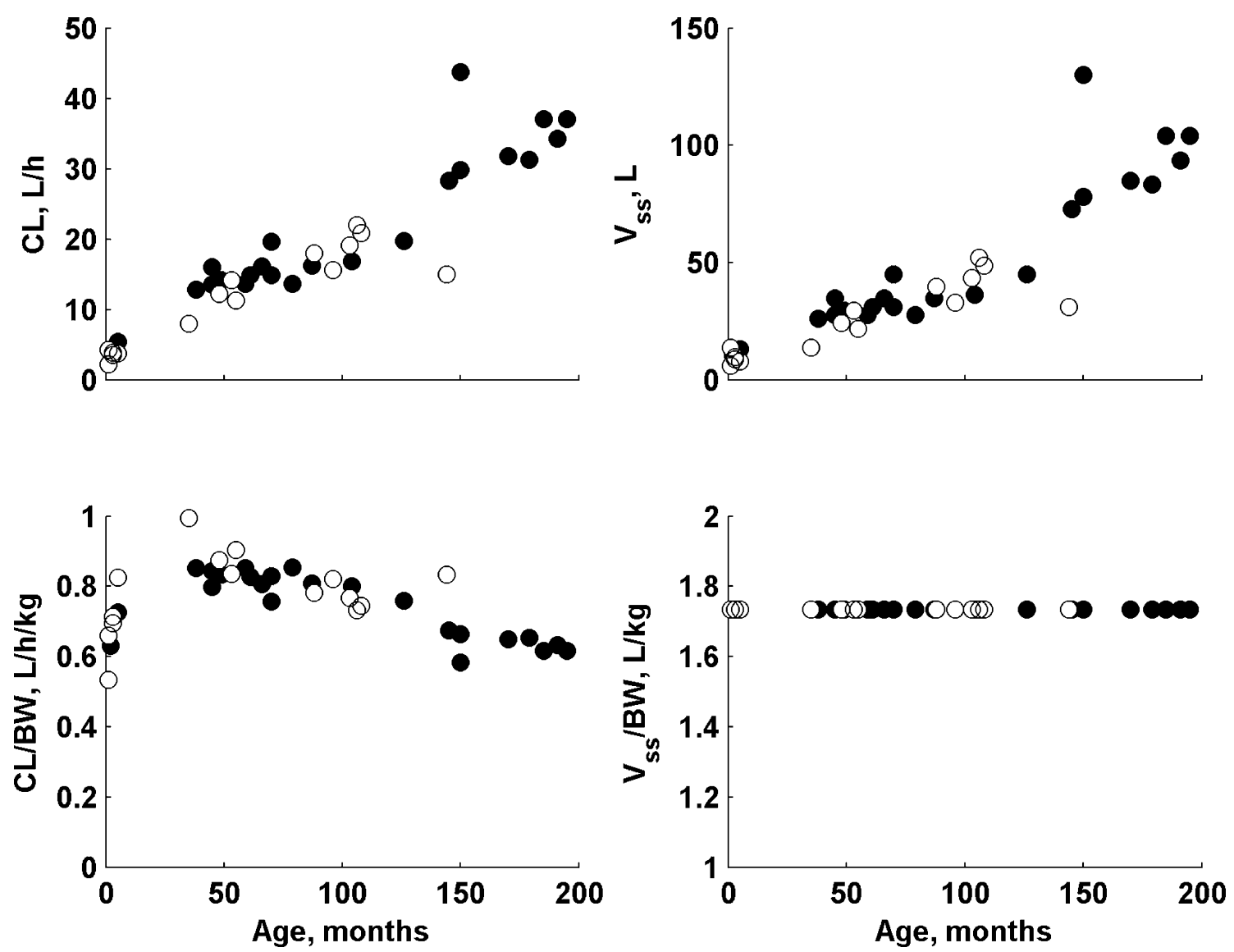

Fig. 3 The individual mean a posteriori values of clearance and volume of distribution (actual and normalized by body weight) in relation to patient age. Closed symbols denote males and open symbols denote females

\section{Discussion}

A population pharmacokinetic model was developed based on the data obtained from PICU in critically ill patients undergoing prolonged infusion. The available small group of patients with wide age and weight range and potential outliers enforced the use of a fully Bayesian approach with informative prior information on the PK parameters to increase the stability of the model developed. The priors were elucidated from one study involving a pooled analysis of various DEX data obtained in children [34].

An interesting phenomenon from the point of view of the pharmacokinetic properties of DEX is increasing clearance during infusion of the drug reported in critically ill adult patients in the ICU. This observation was noted in a study by Iirola et al. [5], in which 13 critically ill patients were treated with constant infusion rate of DEX for the first $12 \mathrm{~h}$. After the first $12 \mathrm{~h}$, the infusion rate of DEX was titrated between 0.1 and $2.5 \mathrm{mg} / \mathrm{kg} / \mathrm{h}$ by using a predefined dose levels to maintain sedation within the range between 0 and 3 on the Richmond Agitation-Scale Sedation. DEX infusion was continued as long as required to a maximum of 14 days. The authors explain this more than two-fold increase in drug clearance by general improvement in the physiological condition of the patients and improvement in liver flows [5]. Our data show moderate evidence of a clinically significant increase in clearance $(\operatorname{Pr}=0.62)$ at the second (post infusion end) occasion. Addressing the literature observation in adults, it is reasonable to conclude that this phenomenon is likely to occur in children.

There is also a strong evidence of an increased volume of the central compartment after infusion cessation. That is very likely due to an unavoidable phenomenon that during the routine administration of the drug, there is a moment when the drug enters the bloodstream despite the end of infusion, as a consequence of its presence in the drug delivery lines. Thus, we observe higher DEX concentrations than expected which in consequence leads to increased value of $V_{1}$. This increased value of $V_{1}$ translates to the increased half-life of the alpha-phase and decreased elimination rate of DEX few minutes upon infusion discontinuation. Also other explanations cannot be excluded, such as altered cardiac index during the recovery after anesthesia, which can alter perfusion rates to tissues and leads to higher $V_{1}$ estimates $[35,36]$. 
In this work, the presence of outliers in the data was handled by assuming a robust t-distribution of residuals. Our approach was different from the one already presented for DEX [37]. In the cited work authors used a finite mixture as the residual error model. Nevertheless, this particular approach could not be used for our dataset as it required unrealistic assumption of an additive residual error model and one compartment disposition model.

The personalized therapy requires the knowledge of drugs pharmacokinetics and factors affecting inter-individual variability in drug response [38]. The elucidation of those factors seems to be particularly important in the pediatric population treated in PICU [39]. We think that the use of Bayesian inference approach might be an effective tool in addressing the often asked questions on the most likely differences between the population of patients investigated and the one that was used to support the current dosing paradigm. This post-data questions are often present when analyzing observational data and can effectively be addressed using Bayesian theory.

In conclusion, a population PK model was successfully developed to describe the time course and variability of dexmedetomidine in PICU patients using allometric principles and clearance maturation model. The disease status described by PRISM score, duration of infusion, and sex were not found to be independent significant covariates in this study. A 1.5-fold increase in the volume of distribution after infusion cessation was observed. There were also some evidences on increased clearance, however, more data is needed to fully confirm clinical significance of this phenomenon.

Supplementary material is available and includes mass spectrometry settings, trace plots of model parameters along the MCMC chain's length, details on selection of fraction parameters $\left(f_{P}\right)$, goodness-of-fit plots, ETA plots, and WinBUGS/Matlab codes of the used models.

Acknowledgments This project was supported by the Grant 2015/17/ B/NZ7/03032 founded by the Polish National Science Centre. We would like to thank Emilia Daghir-Wojtkowiak for her helpful comments.

Open Access This article is distributed under the terms of the Creative Commons Attribution 4.0 International License (http://creative commons.org/licenses/by/4.0/), which permits unrestricted use, distribution, and reproduction in any medium, provided you give appropriate credit to the original author(s) and the source, provide a link to the Creative Commons license, and indicate if changes were made.

\section{References}

1. Allegaert K, Olkkola KT, Owens KH, Van de Velde M, de Maat MM, Anderson BJ (2014) Covariates of intravenous paracetamol pharmacokinetics in adults. BMC Anesthesiol 14:77
2. de Wildt SN (2011) Profound changes in drug metabolism enzymes and possible effects on drug therapy in neonates and children. Expert Opin Drug Metab Toxicol. 7:935-948

3. Smits A, Kulo A, de Hoon JN, Allegaert K (2012) Pharmacokinetics of drugs in neonates: pattern recognition beyond compound specific observations. Curr Pharm Des 18:3119-3146

4. Hoy SM, Keating GM (2011) Dexmedetomidine: a review of its use for sedation in mechanically ventilated patients in an intensive care setting and for procedural sedation. Drugs. 71:14811501

5. Iirola T, Ihmsen H, Laitio R, Kentala E, Aantaa R, Kurvinen JP, Scheinin M, Schwilden H, Schüttler J, Olkkola KT (2012) Population pharmacokinetics of dexmedetomidine during long-term sedation in intensive care patients. Br J Anaesth 108:460-468

6. Alexopoulou C, Kondili E, Diamantaki E, Psarologakis C, Kokkini S, Bolaki M, Georgopoulos D (2014) Effects of dexmedetomidine on sleep quality in critically ill patients: a pilot study. Anesthesiology 121:801-807

7. Nelson LE, Lu J, Guo T, Saper CB, Franks NP, Maze M (2003) The alpha2-adrenoceptor agonist dexmedetomidine converges on an endogenous sleep-promoting pathway to exert its sedative effects. Anesthesiology 98:428-436

8. Weinhouse GL, Schwab RJ (2006) Sleep in the critically ill patient. Sleep 29:707-716

9. Sanders RD, Xu J, Shu Y, Januszewski A, Halder S, Fidalgo A, Sun P, Hossain M, Ma D, Maze M (2009) Dexmedetomidine attenuates isoflurane-induced neurocognitive impairment in neonatal rats. Anesthesiology 110:1077-1085

10. Mason KP, Lerman J (2011) Review article: dexmedetomidine in children: current knowledge and future applications. Anesth Analg 113:1129-1142

11. Schmidt AP, Valinetti EA, Bandeira D, Bertacchi MF, Simões CM, Auler JO (2007) Effects of preanesthetic administration of midazolam, clonidine, or dexmedetomidine on postoperative pain and anxiety in children. Paediatr Anaesth 17:667-674

12. Yuen VM, Hui TW, Irwin MG, Yuen MK (2008) A comparison of intranasal dexmedetomidine and oral midazolam for premedication in pediatric anesthesia: a double-blinded randomized controlled trial. Anesth Analg 106:1715-1721

13. Sheta SA, Al-Sarheed MA, Abdelhalim AA (2014) Intranasal dexmedetomidine vs midazolam for premedication in children undergoing complete dental rehabilitation: a double-blinded randomized controlled trial. Paediatr Anaesth 24:181-189

14. Arcangeli A, D'Alò C, Gaspari R (2009) Dexmedetomidine use in general anaesthesia. Curr Drug Targets 10:687-695

15. Shehabi Y, Botha JA, Ernest D, Freebairn RC, Reade M, Roberts BL, Seppelt I, Weisbrodt L (2010) Clinical application, the use of dexmedetomidine in intensive care sedation. Crit Care Shock. 13:40-50

16. Tobias JD, Berkenbosch JW (2004) Sedation during mechanical ventilation in infants and children: dexmedetomidine versus midazolam. South Med J 97:451-455

17. Lunn DJ, Best N, Thomas A, Wakefield J, Spiegelhalter D (2002) Bayesian analysis of population PK/PD models: general concepts and software. J Pharmacokinet Pharmacodyn 29:271-307

18. Mu S, Ludden TM (2003) Estimation of population pharmacokinetic parameters in the presence of non-compliance. J Pharmacokinet Pharmacodyn 30:53-81

19. Gurrin LC, Moss TJ, Sloboda DM, Hazelton ML, Challis JR, Newnham JP (2003) Uising WinBUGS to fit nonlinear mixed models with an application to pharmacokinetic modelling of insulin response to glucose challenge in sheep exposed antenatally to glucocorticoids. J Biopharm Stat 13:117-139

20. Stickland MD, Kirkpatrick CM, Begg EJ, Duffull SB, Oddie SJ, Darlow BA (2001) An extended interval dosing method for gentamicin in neonates. J Antimicrob Chemother 48:887-893 
21. Meineke I, Türck D (2003) Population pharmacokinetic analysis of meloxicam in rheumatoid arthritis patients. Br J Clin Pharmacol 55:32-38

22. Isbister GK, Friberg LE, Hackett LP, Duffull SB (2007) Pharmacokinetics of quetiapine in overdose and the effect of activated charcoal. Clin Pharmacol Ther 81:821-827

23. Friberg LE, Isbister GK, Hackett LP, Duffull SB (2005) The population pharmacokinetics of citalopram after deliberate selfpoisoning: a Bayesian approach. J Pharmacokinet Pharmacodyn 32:571-605

24. Kechagia IA, Dokoumetzidis A (2015) Development of a paediatric population pharmacokinetic model for valacyclovir from literature non-compartmental values originating from sparse studies and Bayesian priors: a simulation study. J Pharmacokinet Pharmacodyn 42:237-250

25. Dansirikul C, Morris RG, Tett SE, Duffull SB (2006) A Bayesian approach for population pharmacokinetic modelling of sirolimus. Br J Clin Pharmacol 62:420-434

26. Bartkowska-Śniatkowska A, Bienert A, Wiczling P, RosadaKurasińska J, Zielińska M, Warzybok J, Borsuk A, Tibboel D, Kaliszan R, Grześkowiak E (2016) Pharmacokinetics of sufentanil during long-term infusion in critically ill pediatric patients. J Clin Pharmacol 56:109-115

27. Bienert A, Bartkowska-Sniatkowska A, Wiczling P, RosadaKurasińska J, Grześkowiak M, Zaba C, Teżyk A, Sokołowska A, Kaliszan R, Grześkowiak E (2013) Assessing circadian rhythms during prolonged midazolam infusion in the pediatric intensive care unit (PICU) children. Pharmacol Rep. 65:107-121

28. Carrasco $G$ (2000) Instruments for monitoring intensive care unit sedation. Crit Care 4:217-225

29. Pollack MM, Holubkov R, Funai T, Dean JM, Berger JT, Wessel DL, Meert K, Berg RA, Newth CJ, Harrison RE, Carcillo J, Dalton H, Shanley T, Jenkins TL, Tamburro R, Eunice Kennedy Shriver National Institute of Child Health and Human Development Collaborative Pediatric Critical Care Research Network (2016) The pediatric risk of mortality score: update 2015. Pediatr Crit Care Med. 7:2-9

30. Siluk D, Szerkus O, Struck-Lewicka W, Bartosińska E, Kordalewska M, Bujak R, Borsuk A, Bienert A, Bartkowska-Śniatkowska, Warzybok J, Andrzejczuk L, Wiczling P,
Markuszewski MJ, Nasal A, Kaliszan R (2015) LC-MS/MS method development for pharmacokinetic study of dexmedetomidine in pediatric patients. In: LC-MS/MS method development for pharmacokinetic study of dexmedetomidine in pediatric patients. 26th International Symposium on Pharmaceutical and Biomedical Analysis (PBA 2015), Tbilisi, Georgia

31. US Food and Drug Administration (FDA), Department of Health and Human Services, Guidance for Industry. Bionalytical Method Validation (2001)

32. Prototype PKPD model library for WinBUGS. In: Prototype PKPD model library for WinBUGS (user manual: version 1.2). Metrum Institute

33. Anderson BJ, Holford NH (2008) Mechanism-based concepts of size and maturity in pharmacokinetics. Annu Rev Pharmacol Toxicol 48:303-332

34. Potts AL, Anderson BJ, Warman GR, Lerman J, Diaz SM, Vilo S (2009) Dexmedetomidine pharmacokinetics in pediatric intensive care-a pooled analysis. Paediatr Anaesth 19:1119-1129

35. Hartman JC, Pagel PS, Proctor LT, Kampine JP, Schmeling WT, Warltier DC (1992) Influence of desflurane, isoflurane and halothane on regional tissue perfusion in dogs. Can J Anaesth 39:877-887

36. Keating SC, Kerr CL, Valverde A, Johnson RJ, McDonell WN (2013) Cardiopulmonary effects of intravenous fentanyl infusion in dogs during isoflurane anesthesia and with concurrent acepromazine or dexmedetomidine administration during anesthetic recovery. Am J Vet Res 74:672-682

37. Choi L, Caffo BS, Kohli U, Pandharipande P, Kurnik D, Ely EW, Stein CM (2011) A Bayesian hierarchical nonlinear mixture model in the presence of artifactual outliers in a population pharmacokinetic study. J Pharmacokinet Pharmacodyn 38:613636

38. Nebert DW, Vesell ES (2004) Advances in pharmacogenomics and individualized drug therapy: exciting challenges that lie ahead. Eur J Pharmacol 500:267-280

39. Harnisch L, Shepard T, Pons G, Della Pasqua O (2013) Modeling and simulation as a tool to bridge efficacy and safety data in special populations. CPT Pharmacometrics Syst Pharmacol. 2:e28 